\title{
Intuitive Control Strategies for Teleoperation of Active Catheters in Endovascular Surgery
}

\author{
Alain Devreker ${ }^{a, *}$, Phuong Toan Tran ${ }^{a, *}$, Benoît Rosa ${ }^{a, \dagger}$, Herbert De Praetere $^{b}$, Nicolai Häni $^{c}$, \\ Nele Famaey $^{a}$, Dejan Šatovićc ${ }^{c}$ Paul Herijgers ${ }^{b}$, Jos Vander Sloten ${ }^{a}$, Dominiek Reynaerts ${ }^{a}$, \\ Emmanuel Vander Poorten ${ }^{a}$ \\ a Department of Mechanical Engineering, KU Leuven, BE-3001 Leuven, Belgium \\ E-mail: firstname.lastname@kuleuven.be \\ ${ }^{b}$ Department of Experimental Cardiac Surgery, University Hospital Leuven, BE-3000 Leuven, Belgium \\ ${ }^{c}$ Institute of Mechatronics Systems, Zurich University of Applied Sciences, CH-8401 Winterthur, Switzerland
}

\begin{abstract}
Cardiovascular surgeons increasingly resort to catheter-based diagnostic and therapeutic interventions because of their limited invasiveness. Although these approaches allow treatment of patients considered unfit for conventional open surgery, exposure to radiation and high procedural complexity could lead to complications. These factors motivated the introduction of robotic technology offering more dexterous catheters, enhanced visualization and opening new possibilities in terms of guidance and coordinated control. In addition to improvements of patient outcome, through teleoperated catheter control radiation exposure of surgeons can be reduced. In order to limit surgical workload, intuitive mappings between joystick input and resulting catheter motion are essential. This paper presents and compares two proposed mappings and investigates the benefits of additional visual guidance. The comparison is based on data gathered during an experimental campaign involving 14 novices and 3 surgeons. The participants were asked to perform an endovascular task in a virtual reality simulator presented in the first part of this paper. Statistical results show significant superiority of one mapping with respect to the other and a significant improvement of performance thanks to additional visual guidance. Future work will focus on translating the results to a physical setup for surgical validation, also the learning effect will be analysed more in-depth.
\end{abstract}

Keywords: teleoperation; robotic catheter; mapping; guidance; virtual reality; endovascular surgery

\section{Introduction}

For the past years, endovascular catheterization techniques have been increasingly adopted in cardiac surgery. Their minimally invasive nature is appealing, as they allow treatment of patients that would be considered inoperable with conventional open surgery. This is particularly the case for patients suffering from severe aortic stenosis. Although often associated with high peri-operative risks, this condition can nowadays be treated through catheterization using a transcatheter aortic valve implantation (TAVI) technique. ${ }^{1}$ Depending on the patient anatomy and on the de- gree of stenosis, different vascular access sites can be used to insert the surgical instruments that are necessary for the operation. In current transfemoral approaches, guidewires, sheaths and catheters are successively introduced into the femoral artery, steered within the patient's vasculature and passed through the diseased aortic valve. A stent-mounted bioprosthetic valve is then delivered on-site, in place of the natural stenotic valve. Although transfemoral TAVI reduces rates of both death and rehospitalization by more than $30 \%$ when compared to standard therapy, ${ }^{2}$ such intervention comes with a high procedural complexity. As for any catheter-based intervention, the reduced access to

\footnotetext{
${ }^{*}$ A. Devreker and P.T. Tran contributed equally to this study.

${ }^{\dagger}$ B. Rosa is now with the Department of Cardiovascular Surgery, Boston Children's Hospital, Harvard Medical School, Boston, MA, 02115, USA.
} 


\section{A. Devreker and P.T. Tran}

the patient anatomy not only requires the use of dedicated imaging systems, but also demands an increased dexterity to manipulate the flexible instruments that are inserted deep inside the patient. Conventional imaging systems in endovascular surgery - namely, fluoroscopy - rely on Xray and contrast-agent-driven roadmapping, only providing planar information with possibly overlapping structures. The surgeon thus needs to combine this $2 \mathrm{D}$ information with his/her own mental map of the 3D anatomy. Only through much experience will he/she manage to safely steer the catheter in the complex and deformable 3D environment that is the patient's vasculature. In addition, conventional valve delivery systems, such as the Novaflex+ Transfemoral System (Edwards Lifesciences, Irvine, USA), exhibit limited steering capabilities with manual insertion or rotation of the catheter proximal end. Additional maneuverability is often provided through a 1-DOF (degree of freedom) local bending actuation unit at the catheter distal tip. However, the bending direction of these actuation units varies as the catheter advances and twists itself through the vasculature. As the visualization of the overall twist is limited, it is difficult for the surgeon to account for the catheter spatial orientation when steering it. All aforementioned factors result in a large cognitive overhead for the surgeon, which may increase the risk of intra-operative complications such as plaque disruption, tissue damage and tissue perforation. ${ }^{3,4}$

Continuous advances in robotic technology for catheter-based interventions led to the development of robotic systems that aim to reduce the surgeon's work load by providing more dexterous catheters, enhanced visualization and additional guidance. ${ }^{5,6}$ Various types of local actuation have been investigated to enhance the maneuverability of conventional catheters. These include tendon actuation, ${ }^{7-9}$ magnetic steering, ${ }^{10}$ fluidic actuation ${ }^{11,12}$ and shape memory alloys. ${ }^{13}$ Although the additional DOFs that are provided by such mechanisms enlarge the workspace of the catheter, an adequate control strategy and dedicated user interfaces are needed to manage all these DOFs and limit the surgeon's work load. ${ }^{14}$

Robotic technology could help here to improve the surgeon's situation awareness and allow more intuitive control over the catheter. Robotic systems further allow the surgeon to move away from the operating table and operate without being exposed to damaging radiation. Commercial robotic systems offer ergonomic workstations from which the surgeon can remotely control multi-DOFs active catheters. These systems come with various types of input devices to steer guidewires and catheters through the patient's vasculature. Sensei X and Magellan (Hansen Medical, Mountain View, USA), CorPath (Corindus, Waltham, USA), NIOBE (Stereotaxis, St. Louis, USA) and CGCI (Magnetecs, California, USA) are systems that use buttons, mouses and joysticks, while the Amigo system (Catheter Robotics, Mount Olive, USA) proposes a controller that replicates the handle of conventional catheters. Other input devices include, for instance, the control handle developed by Webster, ${ }^{15}$ which presents two slidable buttons to control each DOF of the steerable catheter, or McCoy's system ${ }^{16}$ which uses a joystick to control the appropriate pair of actuators of the catheter. In all cases, specific motions of the input devices are mapped to individual motions of the guidewires and catheters. Most systems require the surgeon to toggle specific buttons or DOFs of the master device to select the appropriate guidewire/catheter DOFs he/she wants to control. Little attention has been given to mappings that allow coordinated control of multiple catheter DOFs, especially for catheter-based surgical interventions, which require navigating the catheter in constraining environments with limited visual feedback. Coordinated control approaches could potentially shorten the long learning curves that are typically associated with catheter procedures. ${ }^{17}$ However, devising a mapping that allows intuitive and efficient operation of multi-DOFs catheters is nontrivial because of the large kinematic dissimilarity that exists between the input devices and the robotic catheter. Few studies have investigated these matters. Csencsits et $a l .{ }^{18}$ investigated mappings for coordinated control of continuum robots but without focusing on surgical applications. Works by Hendrick et al. ${ }^{19}$ and Majewicz et al. ${ }^{20}$ compared the benefits of task space control over joint space control for concentric tubes and steerable needles, respectively. Depending on the surgical application and the employed instruments, trajectories and surgical gestures vary to a great extent. Therefore, the results of these studies cannot be blindly transferred to the specificities of transfemoral TAVI.

The surgical environment in which the surgeon has to operate during transfemoral TAVI has the following characteristics. The aorta is extending from the femoral arteries up to the aortic heart valve along the cranial direction. The aortic arch gives a globally u-shaped geometry to the aorta, which induces an inversion of the direction in which the catheter advances after crossing this arch. In addition, this particular shape is likely to cause overlapping areas on $\mathrm{X}$-ray images, which could necessitate adjustments of the imaging plane during surgery. According to these considerations, simple mappings that use position or rate control in joint or task space might be insufficient. Due to the large dissimilarities between the input device and the catheter workspaces, position control would require the introduction of a clutching mechanism to decouple the motion between the input master device and the catheter so that the master device can be repositioned when it arrives at the end of its stroke. Such mechanism could reduce the performance of the operator because of master-slave misalignments in tool orientation or camera viewpoint that could occur when unclutching. ${ }^{21}$ On the other hand, rate control reduces the awareness of the catheter actuation state, which would likely increase the surgeon's mental load during the operation. Position or rate control can be implemented in joint or task space. Unlike joint space control, task space control specifies target trajectories of the robot's end-effector (catheter tip) in Cartesian space, which typically and inherently requires coordinated control of multiple joints. Such approach is often found more intuitive 
in case of large kinematic dissimilarity between the master and the slave, as it makes abstraction of this dissimilarity. ${ }^{22}$ However, the task space needs to be appropriately chosen. For instance, a task space defined from a coordinate frame attached to an endoscopic view ${ }^{19}$ might be preferred over a task space defined from the fixed world coordinate frame in which the robot is moving. ${ }^{20}$ The implications of particular choices of spaces are hard to anticipate and it is therefore not always immediately clear which frames to select and which mappings to promote. In addition, preferences can vary according to the surgeon and his/her expertise.

The layout and workspace of the available input device will also have an effect upon the intuitiveness of a particular mapping. Limitations in the workspace of the input device will lead through a particular mapping to limitations at the slave side. Clutching mechanisms can be implemented to overcome such limitations but - as previously mentionned can be found non-intuitive and time-consuming. ${ }^{21}$ Input devices showing large and comparatively similar workspaces to the slave side will offer more flexibility in this regard. Note that the design and selection of appropriate input devices is not within the scope of this paper. For more information on this matter, the reader is referred to, e.g., the work developed by Yoon et al. ${ }^{23}$ Truckai $^{24}$ and Breedveld et al. $^{25,26}$

Preliminary work from the authors compared three different mappings for intuitive and efficient catheter navigation during TAVI. ${ }^{27}$ Each of these mappings was evaluated by inserting a robotic catheter inside an artificial model of the aorta. Although the superiority of one mapping was observed, the experiments were only conducted with a single expert surgeon. In the context of the EU-funded project CASCADE (Cognitive AutonomouS CAtheters operating in Dynamic Environments), this work extends the author's investigations about intuitive control strategies by evaluating two of the previously presented mappings with a larger population including novices and multiple expert surgeons. Only two mappings were retained, as the third one aimed to compensate for the rotation of the catheter about its own axis. Since such rotation generally results in intense contacts with the environment and, subsequently, higher risks of damaging the tissue or dislodging calcified regions of the vessel, it was deemed unsafe by clinicians. The mapping that incorporated this behavior was subsequently not included in this study. Experiments are conducted in a dedicated virtual reality (VR) simulator that has been developed for teleoperation of a virtual robotic catheter inside a virtual 3D model of the vasculature (aorta). Unlike realworld experimental setups, a VR simulator provides a controlled environment in which experiments can be prepared within a short time frame, performed within a chosen - but configurable - scenario and, most importantly, conducted in a reliable, repeatable and measurable fashion. This allows adjusting various parameters, such as the vasculature environment and the catheter mechanical characteristics, without being limited by hardware constraints. In addition, this setup also allows verification of what kinds of visual guidance can simplify the operation of robotic catheters by increasing the situational awareness of the surgeon. To this end, the VR simulator allows not only simulation of conventional real-time fluoroscopic imaging, but can also display a $3 \mathrm{D}$ view of the insertion next to an endoscopic view augmented with visual guiding cues.

The remaining of this paper is organized as follows. Section 2 presents the VR environment that has been developed to simulate catheter-based endovascular interventions, including the physics engine, the joystick master interface and the graphical user interface. Section 3 describes the two mappings that were designed in search for an intuitive control of robotic catheters. This section also presents the visual cues that were devised for each mapping. Section 4 presents the experimental setup and the metrics that were used to assess the different mappings, and discusses the experimental results. Finally, Section 5 ends the paper by drawing conclusions from the present study and sketching possible directions for future work.

\section{Virtual reality simulator for endovascular procedures}

VR simulators provide flexible but controlled environments in which experimental scenarios can be readily adjusted and reliably repeated. Following this rationale, a VR environment has been developed to simulate teleoperated insertions of an active catheter inside a $3 \mathrm{D}$ virtual model of the vasculature. Within this environment, a physics engine (Section 2.1) estimates in real time the catheter shape and displacements resulting from commands that are issued by the operator through the user interface (Section 2.2). The physics engine uses a discrete model of the catheter (Section 2.1.1) combined with a lumped parameter model of the vasculature to estimate the interaction forces between the catheter and its environment (Section 2.1.2). A quasi-static minimum energy argumentation then iteratively computes the resulting catheter shape (Section 2.1.3). The user controls the virtual catheter with a dedicated joystick (Section 2.2.1) and monitors the operation he/she is performing with different intra-operative views that are provided by the VR simulator (Section 2.2.2). The following sections detail the different components of the VR simulator.

\subsection{Physics engine}

\subsubsection{Catheter model}

The catheter is modeled as a series of rigid beams interconnected by torsional springs that represent the catheter intrinsic bending stiffness (Fig. 1). The virtual catheter used during the experiments is a 3 -DOFs catheter with a distal 2-DOFs actuator (Fig. 2). As rotating the catheter about its own axis inside the vasculature can result in severe complications, only insertion/retraction $\rho$ and tip bending (amplitude $\beta$, direction $\delta$ ) were allowed in the experiments that were performed. The simulated catheter has a diameter of 
$6 \mathrm{~mm}$ and the distal 2-DOFs actuator is $50 \mathrm{~mm}$ long. The chosen catheter discretization length is $10 \mathrm{~mm}$, as this allows smoothly representing long catheters while still keeping the computational cost acceptable for interactive realtime applications (see Section 4). The maximum bending angle $\beta$ of the distal actuator is limited to $160^{\circ}$. Finally, in order to replicate a realistic bending behavior inside the vasculature, the flexural rigidity of the virtual catheter was set to $1533.5 \mathrm{Nmm}^{2}$ - the rigidity of a commercially available TactiCath catheter that was identified (St. Jude Medical, St. Paul, USA).

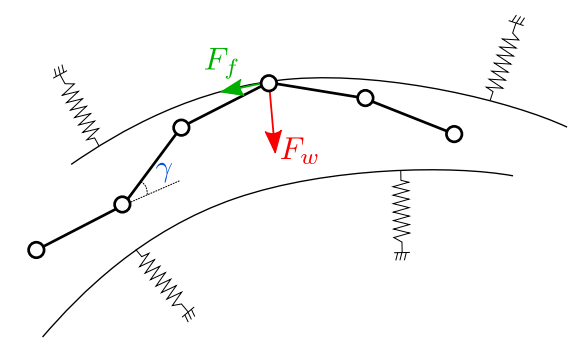

Figure 1. Catheter, vasculature and interaction model of the VR simulator. The catheter is modeled as a series of rigid beams interconnected by torsional springs. The angle $\gamma$ between two consecutive segments relates to the intrinsic bending energy of the catheter. The vessel wall is spanned with linear springs that produce a normal force $F_{w}$ when contact with the catheter occurs. A friction force $F_{f}$ is then also generated at the cathetervessel interface, according to the Coulomb friction model.

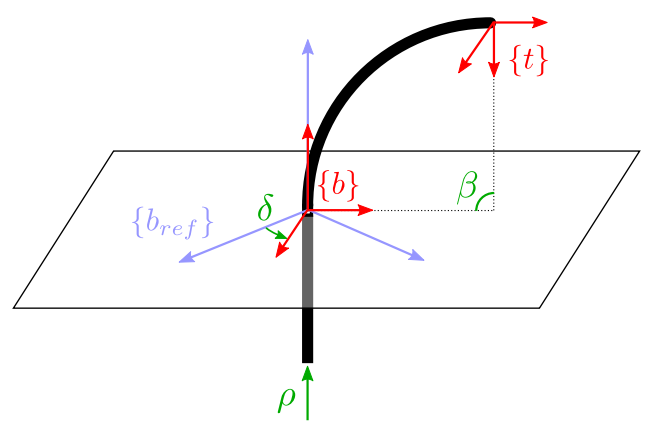

Figure 2. DOFs of the simulated catheter: insertion $\rho$; angle $\delta$ defining the bending direction of the distal actuator as the rotation angle between the reference frame $\left\{b_{\text {ref }}\right\}$ at the base of the distal actuator and the frame $\{b\}$; and angle $\beta$ defining the bending amplitude of the distal actuator. The pose of the catheter tip is defined by the frame $\{t\}$.

\subsubsection{Vasculature model and interaction forces}

Three-dimensional surface meshes of the aorta were segmented from CT scans of seven patients scheduled for TAVI. The patients' CT scans were extracted from a clinical database containing anonymized data. The resulting meshes were used as vascular environment in the VR simulator. The number of geometric primitives for all meshes ranges from 4779 to 5496 triangles.

A lumped material model in which the vessel surface is spanned with orthogonal linear springs accounts for the vessel stiffness during contact interactions with the catheter ${ }^{28}$ (Fig. 1). Although more complex approaches such as finite element (FE) simulations using dedicated material models are able to capture the nonlinear mechanical response of aortic tissue during contact interaction, their computational cost is often prohibitive for real-time applications. To incorporate - to some extent - this nonlinear behavior inside the lumped material model that is used in the VR simulator, the stiffness of the vessel surface was estimated based on FE simulations. To this end, an FE simulation was performed on a volumetric hexahedral mesh (17922 elements, type C3D8R) of an idealized aorta using Abaqus/Standard (version 6.14-1). The dimensions of the idealized geometry $(30 \mathrm{~mm}$ diameter, $2.5 \mathrm{~mm}$ wall thickness, $45 \mathrm{~mm}$ radius of the arch) were chosen to represent realistic aortas. The Holzapfel-Gasser-Ogden material model ${ }^{29}$ was used to account for the nonlinearity and the collagen fiber orientation in the mechanical response of the tissue $\left(C_{10}=0.06 \mathrm{MPa}, D=0, K_{1}=0.27 \mathrm{MPa}, K_{2}=13.4\right.$, $\left.\kappa=0.29, \alpha=45^{\circ}\right)$. A series of nodes on the inner wall, starting from the descending aorta until the ascending aorta, were consecutively loaded with a radial outward force. Force levels of $2.5,5$ and $7.5 \mathrm{~N}$ were successively applied. For each of these force levels and nodes, a linear stiffness was calculated as the ratio between the applied load and the resulting radial displacement. Finally, the obtained stiffnesses were averaged over the different force levels and nodes, yielding a stiffness value of $0.694 \mathrm{~N} / \mathrm{mm}$.

In addition, the force level at which vessel rupture occurs was estimated from the same FE simulation. The rupture force was linearly extrapolated from the maximum circumferential stress occuring in the model during loading, using the rupture stress level defined by Vorp et $a l^{30}$ as a reference. The obtained averaged rupture force is $16.26 \mathrm{~N}$. Note that damage to the vessel will already occur at smaller load levels.

Friction between the catheter and the vessel wall is described by the static Coulomb friction model. A friction coefficient of 0.1 was chosen from the experimental values measured by Kazmierska et $a l .{ }^{31}$

\subsubsection{Catheter-vasculature interaction model}

A quasi-static minimum energy argumentation ${ }^{28,32}$ is used to account for the complex catheter-vessel interaction and predict the changes in the catheter shape result- 
ing from specific positioning commands, such as insertion/retraction, rotation, or bending of active segments. For every command that is sent to the simulator, the catheter bending energy and catheter-vessel interaction forces are estimated and used as inputs to iteratively compute the changes in the catheter shape that would minimize the energy of the entire system (catheter and vessel wall). This interaction model has been extensively validated for navigation of guidewires/catheters in endovascular surgery, ${ }^{28}$ providing sufficiently realistic simulations for the purpose of this study.

\subsection{User interface}

\subsubsection{Joystick for coordinated catheter steering}

The different DOFs of the virtual catheter are controlled by a 4-DOFs haptic joystick that was developed in-house. ${ }^{33}$ Fig. 3 depicts the DOFs of the joystick: the rotational motions $\psi$ (roll), $\theta$ (pitch) and $\phi$ (yaw) orient the joystick's end-effector (handle) about one fixed point in space, while the translational motion $r$ allows moving the handle through this fixed point and in a direction parallel to the longitudinal axis of the handle. The interested reader is referred to Gijbels et al.'s work ${ }^{33}$ for more details on the mechanical design of the joystick.

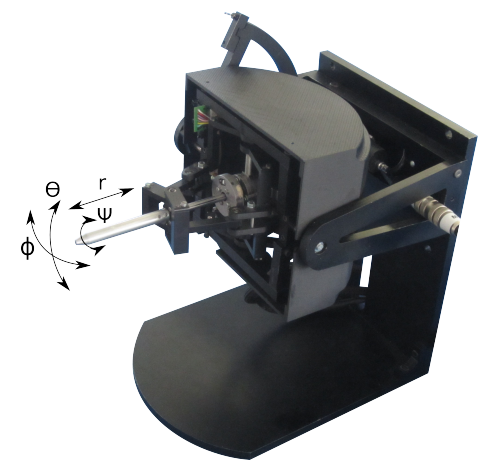

Figure 3. Four-DOFs haptic joystick with a translational DOF $r$ and three rotational DOFs $\psi, \phi$ and $\theta$.

Low-level control of the haptic joystick occurs at $1 \mathrm{kHz}$, which ensures a crisp rendering of desired haptic features. For the specific mappings investigated in this study (see Section 3), virtual walls are generated in the translational $r$-axis and rotational $\theta-$ and $\phi$-axes to make the user aware of, respectively, the catheter insertion speed and the limits on the bending range of the catheter distal actuator. When entering a virtual wall, the user feels a force/torque that is proportional to the penetration distance inside the wall (Figure 4a). For the insertion axis, a virtual wall is positioned on both sides of and close to the joystick's neutral position. The distance along the $r$-axis between both virtual walls is $3 \mathrm{~mm}$ ( $6 \%$ of the $r$-range) and serves as a dead-zone. Hence, the user has to enter the virtual walls to insert or retract the catheter. $\mathrm{He} / \mathrm{she}$ can then relate the insertion speed with the force he/she feels when pushing or pulling the joystick handle. When the user releases the joystick handle, the handle returns to the dead-zone, thus stopping the insertion or retraction. Motions in the translational $r$-axis and in the rotational directions $\psi, \theta$ and $\phi$ are all damped in order to avoid large abrupt movements from the user. The damping forces/torques that are generated by the joystick are proportional to its joint velocities (Figure 4b). The proportional gain $K_{p}$ and differential gain $K_{d}$ used to implement those spring-dampers were tuned with the participation of an expert surgeon to obtain an adequate haptic feeling (see Table 1). Those gains then remained fixed during the study for all participants. Finally, gravity compensation is implemented to improve the transparency of the device.

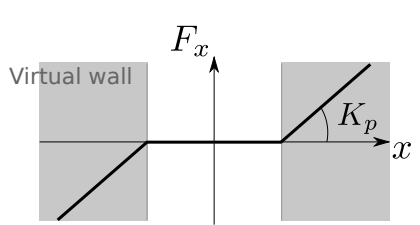

(a)

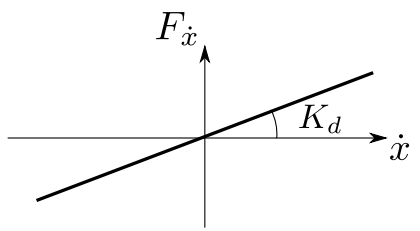

(b)
Figure 4. Haptic cues felt by the user along an $x$-DOF of the joystick, with $x=r, \theta, \phi$ or $\psi$. a) Virtual walls generate a force/torque $F_{x}$ that is proportional (with gain $K_{p}$ ) to the penetration distance inside the wall. b) The damping force/torque $F_{\dot{x}}$ is proportional (with gain $K_{d}$ ) to the joint velocity $\dot{x}$.

Table 1. Proportional and differential gains for haptic cues

\begin{tabular}{c|cc} 
& $K_{p}$ & $K_{d}$ \\
\hline$r$ & $250 \mathrm{~N} / \mathrm{m}$ & $55 \mathrm{Ns} / \mathrm{m}$ \\
$\theta$ & $4 \mathrm{Nm}$ & $0.55 \mathrm{Nms}$ \\
$\phi$ & $4 \mathrm{Nm}$ & $0.55 \mathrm{Nms}$ \\
$\psi$ & $0 \mathrm{Nm}$ & $0.015 \mathrm{Nms}$
\end{tabular}

\subsubsection{Graphical interface}

The VR simulator provides the user with different views on the virtual insertion he/she is performing (Fig. 5). These include a fluoroscopic view, a 3D view of the entire scene and an endoscopic view with visual guiding cues. The former provides a view that is similar to what clinicians observe in clinical practice. The latter two could presumably help the user perform the operation with additional visual information. Although the two latter views are not currently readily available in the operating room, developments of 
new sensing modalities combined with deformable registration techniques ${ }^{34,35}$ or cardiac/respiratory gating ${ }^{36,37}$ have the potential to generate these views in real time during the operation. For instance, to create an endoscopic view, one could use a 6 -DOFs pose sensor (e.g. electromagnetic sensor) embedded within the catheter tip and register the sensor pose to a pre-operative model of the patient's vessel.

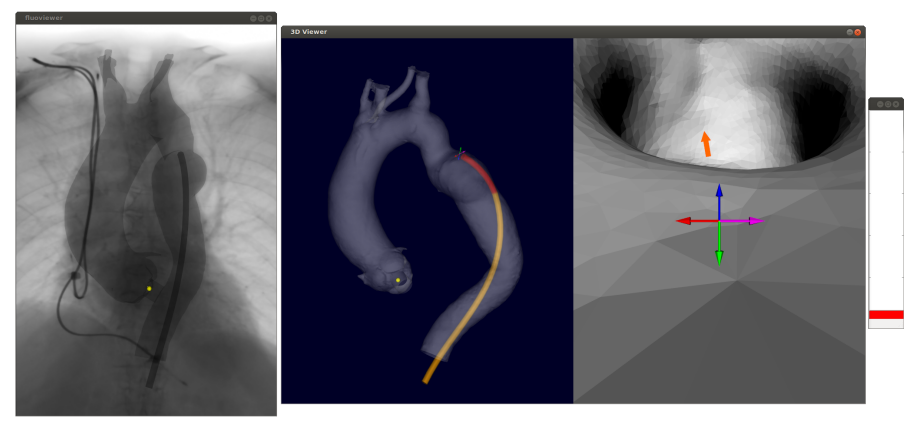

Figure 5. Views provided by the graphical interface. Left: Fluoroscopic view. Right: 3D view and endoscopic view with visual guidance and force gauge.

Synthetic fluoroscopic images are generated using the volumetric data provided by patient CT scans. The volumetric CT data is viewed from the point of view of a virtual camera that uses a composite ray caster to simulate the X-ray physical attenuation process. ${ }^{38}$ The virtual camera is fixed and placed at $1200 \mathrm{~mm}$ from the patient with its image plane parallel to the patient's frontal plane. The simulated $3 \mathrm{D}$ catheter shape is then registered to the camera coordinate frame and projected onto the image plane to generate the $2 \mathrm{D}$ catheter shape in the fluoroscopic image. In real clinical practice, the injection of contrast agent is limited to critical episodes that require a better understanding of the spatial relationship between the catheter and the patient's anatomy. However, in the fluoroscopic images that are generated here, the patient's vasculature is displayed with a continuously enhanced contrast. The rationale is that this study does not aim to assess the ability of the user to detect the catheter and its surroundings, but rather focuses on evaluating his/her ability to navigate the catheter within the virtual vasculature.

A 3D view displays the catheter insertion that is performed by the user within the vessel model. The vessel model is extracted from patient CT scans and shown in transparency so that the catheter that is inserted remains visible. The three-dimensional shape of the catheter is calculated in real time by the physics engine described in Section 2.1. Different color schemes help the user differentiate the active part of the catheter (distal actuator) from its passive part. A SpaceNavigator 3D Mouse (3Dconnexion, Munich, Germany) is put at the user's disposal to adjust the orientation of the $3 \mathrm{D}$ view during the virtual insertion.
Next to the 3D view, an endoscopic view shows the point of view of a virtual camera located at the catheter distal tip. The pose of this virtual camera is directly calculated from the catheter shape that is generated by the physics engine (Section 2.1 and Fig. 2). This endoscopic view makes the user aware of the catheter tip pose with respect to its immediate surroundings, which could facilitate precise control over the pose of the catheter tip and help minimizing the contact interactions with the vessel wall. However, when navigating inside tortuous vessels, the constant change of horizon in the endoscopic view can be source of confusion for the user. ${ }^{14}$ This is emphasized by the specific geometry of the aorta. Additional visual cues are therefore added to the endoscopic view to further guide the user during the virtual insertion. These cues consist in dynamic arrows that help localizing the catheter tip with respect to its surroundings, but also give indications on how the DOFs of the master joystick map to the catheter tip motion. The former is implemented by an arrow that points towards the center of the vessel and appears when the catheter tip moves more than $8 \mathrm{~mm}$ away from the vessel centerline. This has been deemed useful during preliminary tests, e.g. when the catheter tip lays close to the vessel wall. The latter uses a set of arrows whose orientation shows the direction in which the distal actuator is going to bend, and whose length is proportional to the remaining stroke of the actuator. In principle, such arrows should help the user navigate within the vessel, as they directly expose the mapping between the joystick DOFs and the catheter DOFs to the user. The layout of these arrows depends on the control strategy that is currently active (see Section 3). As it becomes difficult to localize the catheter tip with respect to its surroundings when it contacts the vessel wall, these guidance arrows are also displayed in the $3 \mathrm{D}$ view, at the catheter tip.

Finally, a force gauge informs the user about the amplitude of the force that the catheter tip applies on the vessel wall. The interaction force is directly estimated from the model described in Section 2.1. Although the physical model continuously estimates the interaction forces along the entire catheter body, the user is only informed about the tip force, as the catheter tip is the part that would most likely cause damage to the vessel. The maximum amplitude of the force gauge is set to the rupture level estimated in Section 2.1.2.

Note that the interaction force is not fed back by the haptic joystick. The forces that are rendered are only meant to inform the user about the actuation state of the catheter (insertion speed and limits of the bending range). Haptic feedback of the interaction force between catheter and vessel is not provided as at present it is not straightforward to implement such in a reliable and stable manner in complex procedures such as TAVI. 


\section{Control strategies and visual guidance}

Two mappings were developed and investigated in search for a coordinated control strategy that allows intuitive and efficient teleoperation of a robotic catheter. Each of these mappings relates the three catheter DOFs (bending amplitude $\beta$, bending direction $\delta$ and insertion $\rho$ ) described in Section 2.1.1 to some of the four joystick DOFs (angles $\theta, \phi$, $\psi$ and insertion $r$ ) presented in Section 2.2.1. Although the catheter only has three DOFs, the extra joystick DOF gives a larger variety of mappings that can be explored. For both mappings that were designed, the translational DOF $r$ of the joystick commands the insertion $\rho$ of the catheter. For this particular DOF, a rate control with dead-zone is used to avoid the need of implementing a clutching mechanism. This is expressed as

$$
\dot{\rho} \propto \begin{cases}\left(r-r_{0, u}\right) & \text { if } r>r_{0, u} \\ \left(r-r_{0, l}\right) & \text { if } r<r_{0, l} \\ 0 & \text { otherwise }\end{cases}
$$

where $\propto$ denotes a proportional relation and $r_{0, l}$ and $r_{0, u}$ are respectively the lower and upper bounds of the deadzone around the zero position $r_{0}$ of the translational DOF $r$. Any involuntary insertion or retraction that could occur when the user releases the joystick is prevented by the spring-damper with dead-zone that implements this rate control (see Section 2.2.1). The mappings that are here investigated thus only differ in the way the distal actuator of the catheter is controlled by the rotational DOFs of the joystick. Both mappings were designed to increase the user's awareness about the current bending state of the catheter distal actuator.

\subsection{Direct control}

The direct control mapping was designed so that the user has the impression to hold the catheter in his/her hands and can steer it using gestures that are similar to those used for rigid laparoscopic instruments. With this mapping, the direction of the joystick motion directly maps to the direction of the catheter tip motion expressed in the coordinate frame $\{t\}$ rigidly attached to the catheter tip (see Fig. 2). When the catheter twists about its axis, this frame $\{t\}$ will follow and rotate accordingly. This direct mapping can be expressed by the following equations:

$$
\left\{\begin{array}{l}
\delta=\arctan 2\left(-\frac{\theta}{\theta_{\max }} ;-\frac{\phi}{\phi_{\max }}\right) \\
\beta=\beta_{\max } \sqrt{\left(-\frac{\theta}{\theta_{\max }}\right)^{2}+\left(-\frac{\phi}{\phi_{\max }}\right)^{2}}
\end{array}\right.
$$

where the subscript max indicates the maximal angle that can be reached within the joystick workspace. The bending direction $\delta$ depends on the location of the joystick handle on the surface parameterized by angles $\theta$ and $\phi$ for the current $r$ value, while the bending amplitude $\beta$ relates to the distance of the current joystick position to the joystick center position. The minus sign in Eq. 2 expresses that the resulting bending motion of the catheter tip is inverted with respect to the user's input so as to mimic laparoscopic settings. The mapping is illustrated in Fig. 6a.

In order to aid the user during the operation, visual guidance in the form of arrows is added to the endoscopic view (Fig. 6b). These vertical and horizontal arrows point towards the direction in which the catheter tip will bend when moving the joystick handle along the $\theta$ and $\phi$ axes, respectively. The length of these arrows is proportional to the remaining range of motion allowed along the respective axes. As mentioned in Section 2.2.2, an additional arrow (orange arrow in Fig. 6b) guide the user during the navigation by pointing towards the center of the vessel.

\subsection{Bending plane and amplitude control}

Whereas the direct control mapping reproduces to some extent gestures of laparoscopic procedures, this mapping presents more similarities with gestures that surgeons perform during conventional TAVI. As depicted in Fig. 6c, the bending direction $\delta$ is mapped to the joystick angle $\psi$, while the bending amplitude $\beta$ is controlled by the joystick angle $\theta$. This is formulated as:

$$
\left\{\begin{array}{l}
\delta=2 \psi \\
\beta=\beta_{\max }\left(\frac{\theta_{\max }-\theta}{2 \theta_{\max }}\right)
\end{array}\right.
$$

Such mapping decouples the bending direction from the bending amplitude. This could facilitate precise adjustment of the bending direction while keeping the bending amplitude constant. Note that, independent of this decoupling, the user can still control the bending DOFs in a coordinated manner.

The visual guidance for this mapping consists of a single arrow whose orientation shows the direction in which the catheter will bend when moving the joystick along the $\theta$ axis. The length of the arrow is proportional to the remaining bending range associated with this axis (Fig. 6d). The orientation of the arrow depends on the rotation angle about the $\psi$ axis. As for the direct control mapping, additional guidance is provided by an arrow pointing towards the center of the vessel.

\section{Experiments}

\subsection{Software implementation}

The VR simulator has been implemented within the framework of the CASCADE software platform. Developed by the CASCADE project, this platform aims not only to facilitate the software developments that are necessary for complex surgical robotic systems, but also to serve as an interface to provide intuitive access to a vast amount of 


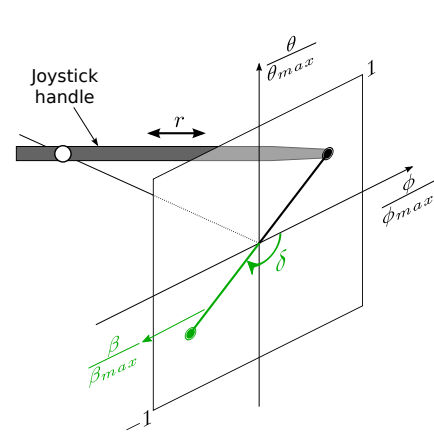

(a)

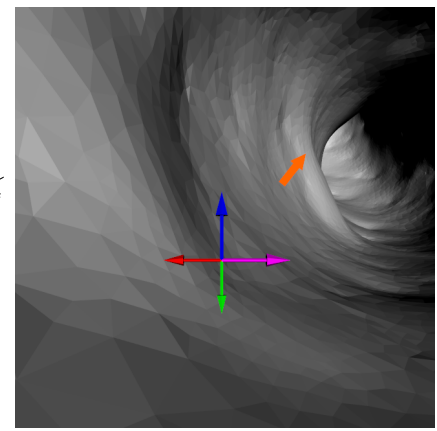

(b)

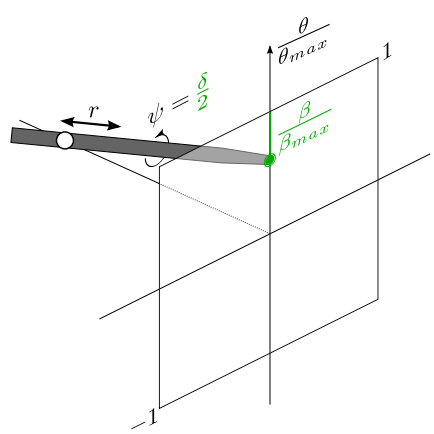

(c)

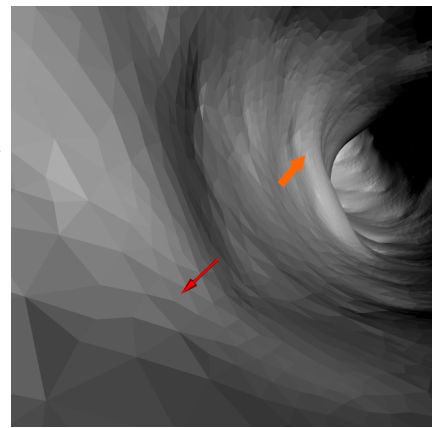

(d)

Figure 6. Schematic description of the investigated mappings. For both mappings, the joystick translational DOF $r$ is mapped to the catheter insertion/retraction using a rate control. a) For the direct mapping, the joystick DOFs $\theta$ and $\phi$ are mapped to the bending DOFs $\beta$ and $\delta$ of the catheter. b) Endoscopic view with visual guidance for the direct mapping. c) For the bending plane and amplitude mapping, the joystick DOFs $\psi$ and $\theta$ are mapped to the bending DOFs $\beta$ and $\delta$ of the catheter. d) Endoscopic view with visual guidance for the bending plane and amplitude mapping.

data generated by various sensors, algorithms and user interfaces. The platform relies on ROS $^{39}$ for messaging and non-realtime tasks, while hard real-time tasks are handled by OROCOS. ${ }^{40,41}$ Graphical interfaces use Qt and VTK ${ }^{42}$ for user interaction and visualization.

Within the physics engine (Section 2.1), computations of the catheter/vessel interactions are parallelized using OpenMP. ${ }^{43}$ This is necessary due to the iterative nature of the interaction model that requires the execution of a high number of collision queries to compute the contact forces between the catheter and the vasculature. Although the computional cost of such queries mainly depends on the resolution of the catheter and vessel models, parallelization of these queries was required to reach interactive update rates. An update rate of $26 \mathrm{~Hz}$ was obtained on a 64-bits machine with an Intel Core i7-4910MQ CPU and 16Gb of RAM for a vessel mesh of 6000 triangles and 53 catheter nodes separated by $10 \mathrm{~mm}$ rigid segments.

\subsection{Experimental conditions}

An overview of the experimental setup is shown in Fig. 7. Participants take place in front of two screens that display the different views provided by the VR environment. The haptic joystick is positioned on a table at the user elbow level. The $3 \mathrm{D}$ mouse that controls the orientation of the $3 \mathrm{D}$ view is placed next to the joystick. Before starting the experiment, the relative position of the joystick and 3D mouse is adjusted depending on the user's handedness.

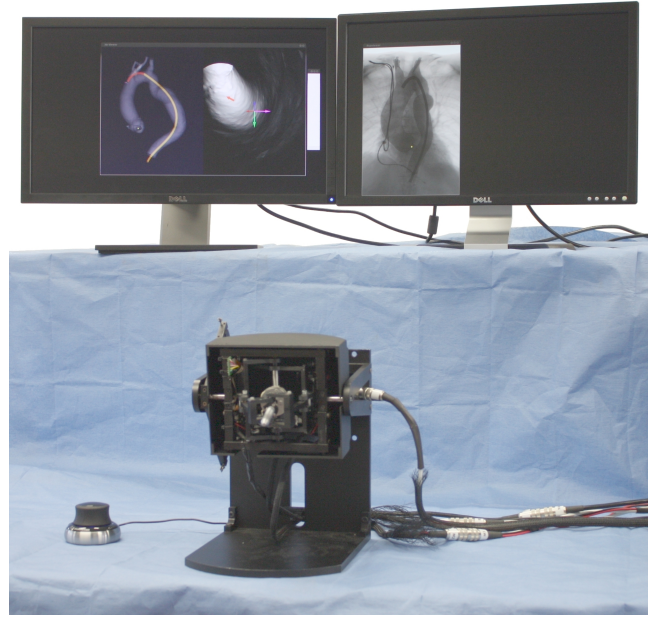

Figure 7. Experimental setup, which comprises a haptic joystick, a 3D mouse and two screens displaying the VR simulator.

An experimental task has been designed to investigate how the mapping between the joystick and the catheter DOFs and the addition of visual guidance influence the performance of different users when steering a robotic catheter. The task consisted in virtually inserting a robotic catheter inside a 3D model of an aorta using the VR simulator described in Section 2. Users started with the catheter located at the level of the abdominal aorta. They had to steer the catheter along the aorta, cross the aortic arch and finally position the catheter tip at the center of the aortic annulus. A yellow sphere presented on all views helped the users locating the annulus central point (see Fig. 5). Users were instructed to perform the insertion fast and smoothly, but more importantly to avoid as much as possible contact 
between the catheter tip and the vessel wall, as excessive contact interactions can lead to plaque disruption, tissue damage and/or tissue perforation in real surgery.

All users were asked to test the two mappings defined in Section 3 without visual guidance (i.e. with only the fluoroscopic view) and with visual guidance (i.e. with fluoroscopic, 3D and endoscopic views). This led to four different test modes that are labelled following Table 2. For each user, the order in which the test modes are executed is randomized first based on the mapping condition, then based on the visual guidance condition for the selected mapping. The first selected mapping is thus tested with and without guidance (in a random order) before testing the second one. The trials for each mode included a learning phase of three insertions. After the learning phase of each mode, users were asked to perform five insertions. For each insertion, the starting position of the catheter within the abdominal aora was randomized, and the aorta geometry was randomly chosen between seven different meshes. For the learning phase, only the starting position of the catheter was randomized.

A brief slideshow was presented to all users so that all received an identical set of instructions. The content of this slideshow gave a brief introduction to TAVI, exposed the related difficulties, and described the experimental setup, the different mappings and the task to perform. The start of the experiment was orally indicated to the user by the supervising person. Once the catheter tip had reached the target position, the user indicated the end of the task to the supervising person, who stopped the recording. After each test mode, the user was asked to fill in a questionnaire evaluating the task workload. ${ }^{44}$ The user was also invited to provide comments on the test modes and on the setup itself.

Table 2. Numbering of test modes

\begin{tabular}{r|cc} 
& \multicolumn{2}{|c}{ Guidance } \\
& Without & With \\
\hline Direct control & 1 & 2 \\
Bending plane and amplitude control & 3 & 4
\end{tabular}

\subsection{Assessment of performance}

In order to assess the performance of the participants, different metrics were recorded during the experiments. These metrics were defined in collaboration with an expert TAVI surgeon so as to capture as much as possible clinically relevant parameters. Care was taken to ensure that metrics were independent from the individual experimental conditions (type of mapping and visual guidance). Some of the selected metrics, such as completion time, path length, smoothness of the path and estimation of tissue damage are common for surgical skills assessment, ${ }^{45-47}$ others such as interaction force or number of pullbacks are less common. All metrics are evaluated for a single catheter insertion.

- $T_{f}$ is the time required to perform the catheter insertion. This is measured from the moment the user starts inserting the catheter until the moment that he/she informs the supervising person to have reached the target location.

- $L_{p}$ is the relative path length followed by the catheter tip. It is defined as the distance covered by the catheter tip divided by the length of the centerline of the aorta $3 \mathrm{D}$ model.

- $\eta_{S A L}$ is the smoothness of the path followed by the catheter tip. Using the spectral arc length method, it is defined by the spectral arc of a speed profile spectrum. ${ }^{48}$ Correlated to the expertise of endovascular surgeons this method is robust, adimensional, speed- and time-independent. ${ }^{49}$ The result is a negative number with an absolute value closer to zero for smoother movements.

- $T_{c}$ corresponds to the percentage of the time during which the catheter tip is in contact with the vessel wall.

- $F_{\text {mean }}$ is the mean force applied on the vessel wall by the catheter tip.

- $F_{m e d}$ is the median force applied on the vessel wall by the catheter tip.

- $F_{\max }$ is the maximal force applied on the vessel wall by the catheter tip.

- $N_{t}$ represents the number of times the catheter tip touches the vessel wall. It could be interpreted as a safety criterion since every touch can disrupt plaques or damage the vessel tissue.

- $N_{p}$ is the number of pull-backs during the insertion. It could be an indication of the hesitation and/or difficulty the user experienced when steering the catheter.

\subsection{Results}

In total 14 novices, i.e. people with technical background and various extents of experience in handling haptic devices for robotic surgery but without any surgical experience, and 3 expert TAVI surgeons, i.e. endovascular surgeons who performed more than 50 surgical procedures, participated to the experimental campaign. All of them managed to steer the catheter tip to the aortic annulus center. In total 610 insertions were recorded, learning phase included. After removing improper experiments (6 in total) - such as experiments where a simulation error occurred or the subject was interrupted by an external factor - and excluding the learning phase (226 in total), 378 experiments remained.

After these removals, some users only had 4 recorded insertions left. Therefore, only the first 4 experiments after the learning phase were considered for each mode and for each user. As a result, from the original 610 experiments performed, 272 data sets remained, corresponding to 17 users performing 4 insertions for the 4 different modes.

A statistical analysis has been performed on the con- 
sidered data. Normality of the data could not be assumed, as visually confirmed by a QQ-plot and tested with a Shapiro-Wilk normality test (p-values $<10^{-4}$ ). Nonparametric tests were therefore applied to analyze the data. The Friedman test - similar to the parametric repeated measures ANOVA test - was chosen to assess the statistical difference between the four modes for all continuous metrics. A statistical difference was found for all metrics (p-value < 0.05). A post-hoc Mann-Whitney U-test was then performed to identify any statistical difference between pairs of mode, metric by metric. A Bonferroni correction was applied to compensate for repeated testing. This test was performed separately for novices and experts.

The p-values $p_{U}^{i-j}$ resulting from the post-hoc MannWhitney U-tests between mode $i$ and mode $j$ are summarized in Table 3. Table cells are marked with $\mathrm{N}$ and/or E if a statistically significant difference after Bonferroni correction ( $p$-value $<0.01$ ) has been found for Novices and Experts, respectively. Tables 4 and 5 provides the interquartile ranges (IQR) and medians for experts and novices for all modes. From Table 3 , the maximal force $F_{\max }$ appears to be of smaller importance compared to the mean force $F_{\text {mean }}$ or the median force $F_{\text {med }}$. Indeed, large peak forces appeared occasionally (once or twice per simulation) at a more or less arbitrary time and catheter position. Since such force appeared as large peaks the force amplitude was not considered as a reliable and consistent metric. The number of pullbacks $N_{p}$ was also judged not to convey relevant information, as the interquartile ranges and medians of this metric are respectively equal to 1 and 0 for all modes. For this reason, this metric will not be considered either in the next sections.

\begin{tabular}{l|cccc} 
Metrics & Mode 1 & Mode 2 & Mode 3 & Mode 4 \\
\hline$T_{f}$ & $\mathbf{2 2 / 1 3}$ & $23.51 / \mathbf{9 . 9 1}$ & $50.30 / 19.08$ & $44.22 / 60.00$ \\
$L_{p}$ & $0.83 / 0.85$ & $\mathbf{0 . 3 2} / \mathbf{0 . 1 5}$ & $1.42 / 1.20$ & $0.63 / 0.65$ \\
$\eta_{S A L}$ & $46.9 / 31.5$ & $\mathbf{4 0 . 6} / \mathbf{1 1 . 8}$ & $98.2 / 96.2$ & $112.6 / 142.4$ \\
$T_{c}$ & $0.17 / 0.27$ & $\mathbf{0 . 1 0} / \mathbf{0 . 0 5}$ & $0.22 / 0.27$ & $0.20 / 0.18$ \\
$F_{\text {mean }}$ & $0.25 / 0.35$ & $\mathbf{0 . 0 3} / \mathbf{0 . 0 2}$ & $0.28 / 0.59$ & $0.05 / 0.04$ \\
$F_{\text {med }}$ & $0.18 / 0.31$ & $\mathbf{0 . 0 0} / \mathbf{0 . 0 0}$ & $0.17 / 0.65$ & $\mathbf{0 . 0 0} / \mathbf{0 . 0 0}$ \\
$F_{\text {max }}$ & $\mathbf{1 . 4 9} / \mathbf{1 . 5 5}$ & $1.95 / 1.95$ & $1.60 / 2.22$ & $3.31 / 2.94$ \\
$N_{t}$ & $4 / 3$ & $\mathbf{3} / \mathbf{2}$ & $6.5 / 10.75$ & $7 / 6.75$ \\
$N_{p}$ & $1 / \mathbf{0}$ & $\mathbf{0} / \mathbf{0}$ & $1 / 1$ & $1 / \mathbf{0}$
\end{tabular}

Table 5. Medians for all metrics and for the different modes. Each cell contains the median for novices followed by the value for experts. The best medians for novices and experts are highlighted in bold.

\begin{tabular}{l|cccc} 
Metrics & Mode 1 & Mode 2 & Mode 3 & Mode 4 \\
\hline$T_{f}$ & $45.88 / 31.96$ & $\mathbf{3 8 . 9 7 / 3 0 . 0 1}$ & $60.99 / 42.06$ & $73.32 / 62.66$ \\
$L_{p}$ & $2.09 / 2.49$ & $\mathbf{1 . 8 6 / 1 . 7 2}$ & $2.70 / 2.64$ & $2.32 / 2.25$ \\
$\eta_{S A L}$ & $\mathbf{- 5 7 . 1 / - 4 9 . 7}$ & $\mathbf{- 5 7 . 1 / - 4 1 . 2}$ & $-109.0 /-68.2$ & $-83.0 /-106.7$ \\
$T_{c}$ & $0.64 / 0.66$ & $\mathbf{0 . 1 2} / \mathbf{0 . 0 6}$ & $0.66 / 0.77$ & $0.17 / 0.31$ \\
$F_{\text {mean }}$ & $0.27 / 0.40$ & $\mathbf{0 . 0 3} / \mathbf{0 . 0 2}$ & $0.32 / 0.41$ & $0.04 / 0.04$ \\
$F_{\text {med }}$ & $0.13 / 0.21$ & $\mathbf{0 . 0 0} / \mathbf{0 . 0 0}$ & $0.15 / 0.34$ & $\mathbf{0 . 0 0} / \mathbf{0 . 0 0}$ \\
$F_{\text {max }}$ & $1.89 / 2.16$ & $\mathbf{1 . 8 7} / \mathbf{1 . 5 8}$ & $2.49 / 2.72$ & $2.25 / 2.55$ \\
$N_{t}$ & $7 / 8$ & $\mathbf{5 / 3}$ & $8 / 8$ & $7 / 10$ \\
$N_{p}$ & $0 / 0$ & $0 / 0$ & $0 / 0$ & $0 / 0$ \\
& & & &
\end{tabular}

\subsection{Discussion}

This section presents and discusses the major outcomes of this study.

Table 3. Results of Mann-Whitney U-Test accross test modes, $\mathrm{N}$ and/or $\mathrm{E}$ stands for statistically significant difference after 4.5.1. Guidance Bonferroni correction ( $\mathrm{p}$-value $<0.01$ ) for Novices and Experts respectively. Indices indicate the superior test mode number.

\begin{tabular}{l|cccccc} 
Metrics & $p_{U}^{1-2}$ & $p_{U}^{1-3}$ & $p_{U}^{1-4}$ & $p_{U}^{2-3}$ & $p_{U}^{2-4}$ & $p_{U}^{3-4}$ \\
\hline$T_{f}$ & - & - & $\mathrm{N}_{1}+\mathrm{E}_{1}$ & $\mathrm{~N}_{2}$ & $\mathrm{~N}_{2}+\mathrm{E}_{2}$ & - \\
$L_{p}$ & $\mathrm{~N}_{2}+\mathrm{E}_{2}$ & $\mathrm{~N}_{1}$ & - & $\mathrm{N}_{2}+\mathrm{E}_{2}$ & $\mathrm{~N}_{2}+\mathrm{E}_{2}$ & - \\
$\eta_{S A L}$ & - & $\mathrm{N}_{1}$ & $\mathrm{~N}_{1}+\mathrm{E}_{1}$ & $\mathrm{~N}_{2}+\mathrm{E}_{2}$ & $\mathrm{~N}_{2}+\mathrm{E}_{2}$ & - \\
$T_{c}$ & $\mathrm{~N}_{2}+\mathrm{E}_{2}$ & - & $\mathrm{N}_{4}+\mathrm{E}_{4}$ & $\mathrm{~N}_{2}+\mathrm{E}_{2}$ & $\mathrm{E}_{2}$ & $\mathrm{~N}_{4}+\mathrm{E}_{4}$ \\
$F_{\text {mean }}$ & $\mathrm{N}_{2}+\mathrm{E}_{2}$ & - & $\mathrm{N}_{4}+\mathrm{E}_{4}$ & $\mathrm{~N}_{2}+\mathrm{E}_{2}$ & $\mathrm{E}_{2}$ & $\mathrm{~N}_{4}+\mathrm{E}_{4}$ \\
$F_{\text {med }}$ & $\mathrm{N}_{2}+\mathrm{E}_{2}$ & - & $\mathrm{N}_{4}+\mathrm{E}_{4}$ & $\mathrm{~N}_{2}+\mathrm{E}_{2}$ & $\mathrm{~N}_{2}+\mathrm{E}_{2}$ & $\mathrm{~N}_{4}+\mathrm{E}_{4}$ \\
$F_{\text {max }}$ & - & - & - & $\mathrm{N}_{2}+\mathrm{E}_{2}$ & - & - \\
$N_{t}$ & $\mathrm{~N}_{2}+\mathrm{E}_{2}$ & - & - & $\mathrm{N}_{2}+\mathrm{E}_{2}$ & $\mathrm{~N}_{2}+\mathrm{E}_{2}$ & - \\
$N_{p}$ & $\mathrm{~N}_{2}$ & - & - & $\mathrm{N}_{2}+\mathrm{E}_{2}$ & $\mathrm{~N}_{2}$ & -
\end{tabular}

As described in Section 2, the simulated catheter provides an insertion DOF, as well as two distal bending DOFs that allows steering in 3D space. However, the imaging modalities that are currently used intraoperatively only provide $2 \mathrm{D}$ images in which catheter movements that occur out of the image plane are difficult to detect. This can lead to confusion about the actual position of the catheter, and thus to user's hesitation and/or excessive contact between the catheter tip and the vessel wall. Providing the user with additional visual information that aims to guide him/her during the surgery can reduce this ambiguity.

The added value of visual guidance is assessed by comparing modes with the same mapping but different visual interfaces, i.e. by comparing mode 1 with mode 2 , and mode 3 with mode 4 . By doing so, possible detected differences can be considered as independent from the mapping. As seen in Table $3, p_{U}^{1-2}$ and $p_{U}^{3-4}$ for the time of contact $T_{c}$, mean force $F_{\text {mean }}$ and the median force $F_{\text {med }}$ show a statistically significant difference for modes with and without guidance. This statistical difference, as well as
Table 4. Interquartile ranges (IQR) for all metrics and for the different modes. Each cell contains the IQR for novices followed by the value for experts. The smallest IQR for novices and experts are highlighted in bold. 
the medians of the metrics (see Table 5), show that providing surgeons with improved imaging modalities would significantly improve their performance when steering multiDOFs catheters.

\subsubsection{Mappings}

Significant p-values $(<0.05)$ for the Friedman test show that user performance differs for all test modes. This is due not only to the improved awareness provided by visual guidance, but also to the mapping that is used (Table 3 ). As concluded in previous section, steering a multi-DOFs catheter using only the planar images provided by fluoroscopy is complex and requires an adequate mental representation of the patient's anatomy in 3D space, as well as an excellent understanding of the behaviour of the robotic system. When comparing the modes 1 and 3, both without visual guidance, despite a tendency for mode 1 to have better performance (lower medians and IQR values) the difference was not found statistically different. Users were probably not sufficiently acquainted to perform such task using those modes. With increased learning phase and improved knowledge of the task, statistical differences, similar to the comparison of the modes 2 and 4 both with visual guidance, would possibly be observed. Therefore, the mappings are enly compared in modes with visual guidance, i.e. modes 2 and 4 . P-values $p_{U}^{2-4}$ show a significant difference for almost all metrics for novices and experts.

The significant p-values, IQR and medians related to the corresponding metrics show that, with mode 2 , novices performed the task significantly faster and smoother while applying less force on the vessel wall when compared with mode 4 . Time of contact $T_{c}$ and mean force $F_{\text {mean }}$ have p-values of 0.02 and 0.05 respectively which are not in the confidence interval of $1.25 \%$ recalculated with a Bonferroni correction factor. As the Bonferroni correction is considered conservative, the relatively small p-values of those factors should also be taken into account. ${ }^{50,51}$

Regarding experts, better results were expected for modes based on the bending plane mapping as it presents similarities with current surgical gestures. In addition, expert feedback was in favour of a combination of both mappings as the direct mapping is apparently more intuitive than the bending plane mapping while the latter apparently facilitates crossing the aortic arch. However, statistical analysis shows a significant superiority of the direct mapping based on all considered metrics.

In conclusion, both novices and experts performed significantly better with mode 2 than with mode 4 . This contradicts the conclusion of our previous work ${ }^{27}$ where the bending plane mapping seemed to perform better than the direct joint control mapping. This difference could be explained by the different populations that were involved in both studies (one expert vs multiple novices and experts).

\subsubsection{Effect of previous experience}

The performance of experts and novices were compared to evaluate the effect of user experience. A Mann-Whitney Utest across all experiments indicates significant better performances for experts in terms of completion time $T_{f}(\mathrm{p}-$ value $<0.01)$. In addition, when only considering mode 2 , experts were performing better in terms of all metrics.

Based on those statistical results, it is concluded that user experience has a significant positive effect for this experimental campaign. This effect is further increased when using the direct mapping.

In addition, a Friedman test comparing the intersubjects performance within the group of experts showed a statistical significance for all considered metrics. This suggests that experts perform differently under the same conditions. It is further supported by the fact that the surgeons were not using the same views of the VR simulator during the experiments. Two of the three experts were mainly using the endoscopic view locating the catheter within the patient anatomy by identifying anatomical landmarks - identification that novices are not able to perform. The third expert however mentions that the $3 \mathrm{D}$ view was useful to him. This indicates that previous experience and training of the user affects his/her performances and way of performing the gestures. Hence, VR simulation in early training for complex gestures might be very beneficial for training the next generation of surgeon with complex robotic devices.

\subsubsection{Experimental hypotheses and limitations}

As any study, results are to a certain extent influenced by the setup implementation and design choices that were made.

One aspect which shows opportunities for further improvement is the simulation engine and algorithms that were used to model the mechanical interaction between the catheter and the vasculature. While users and experts generally appreciated the employed engine, at some occasions force amplitude was not realistic. These runs were considered as outliers and removed from the analysis, but we recognize that more sophisticated simulation approaches such as e.g. relying on real-time FEM $^{52}$ could possibly allow drawing stronger conclusions.

Also as argued earlier the choice for a certain input device affects to some extent the intuitiveness of the experiments. Whereas the joystick was perceived well by the users and adequate to perform the task at hand, one can expect that alternative joysticks with other configurations, workspaces and/or force ranges could also play a role and somewhat alter the results.

Also, the graphical interface might have influenced the results to some extent. Here, we chose to show simplified fluoroscopic images. The idea was that also novices would be able to interpret what happened in the vasculature. Logically, if more realistic images were used, one can expect that there would be a greater variation between novice and 
expert performance.

Further experiments could help clarifying the effect of the different limitations, although the authors expect that the general trend and main conclusions of this work will likely stand.

\section{Conclusion}

This paper presents intuitive control strategies for teleoperation of active catheters in endovascular surgery using a virtual reality simulator. Experiments are conducted on a TAVI application, where a dedicated simulator is used to model and display the motion of an active endovascular catheter in response to user joystick input commands. An experimental campaign consisting of 14 novices and 3 expert surgeons performing a total of 272 insertions under 4 different test conditions has been conducted to compare the effect of different control strategies and of providing additional visual information. A statistical analysis has been performed to identify differences between the different operation modes.

Statistical results show a significant superiority of the direct control strategy over the bending plane and amplitude control strategy for novices and experts. It has also been shown that outcomes of endovascular surgery using multi-DOFs active catheters will likely improve when enhanced visualization is available. Finally, in line with the expectations, it has been demonstrated that experience with the proposed setup has a significant impact on decision-making and performance. This aspect can be seen between novices and surgeons but also across surgeons with similar level of experience in endovascular surgery.

Future work will be directed towards improvements of the VR simulator setup in order to take finer effects into account. Points of interest include improved mechanical and material properties of the aortic wall, for instance by including real time FEM computations into the simulation framework, ${ }^{52}$ as well as consideration of multi-segment active catheters. Finally, transposing the results to a physical setup for surgical validation will be considered. A particular point of interest is to take vasculature deformation during the cardiac cycle into account in order to generate accurate guidance information and $3 \mathrm{D}$ views in real time. Deformable registration algorithms, ${ }^{34,35}$ as well as movement gating based on the cardiac and respiratory cycles, ${ }^{36,37}$ will be considered for that purpose.

This work showed significant differences between novices and experts, but also between different experts with the same level of experience (i.e. endovascular surgeons). Hence, further studies should be carried out in order to assess more precisely the difference between experts. This result will be particularly interesting to understand the link between training and performance, resulting in new training paradigms in VR environments for the next generation of surgeons. Other procedures where catheters are used to navigate in the intricate vasculature of the patients, such as endovascular neurointervention and transseptal mitral valve repair ${ }^{53}$ deserve further investigation as well.

\section{Acknowledgments}

Research funded by the European Commission's 7th Framework Programme FP7-ICT - Under grant agreement No 601021, EU project CASCADE.

\section{Bibliography}

[1] E. Ferrari and L. von Segesser, "Transcatheter aortic valve implantation (TAVI): state of the art techniques and future perspectives," Swiss Medical Weekly, no. December, pp. 1-11, Dec. 2010.

[2] R. R. Makkar, G. P. Fontana, H. Jilaihawi, S. Kapadia, A. D. Pichard et al., "Transcatheter aortic-valve replacement for inoperable severe aortic stenosis," New England Journal of Medicine, vol. 366, no. 18, pp. 1696-1704, 2012.

[3] A. Aminian, J. Lalmand, and B. El Nakadi, "Perforation of the descending thoracic aorta during transcatheter aortic valve implantation (TAVI): An unexpected and dramatic procedural complication," Catheterization and Cardiovascular Interventions, vol. 77, no. 7, pp. 1076-1078, jun 2011.

[4] J. B. Plotkin, R. J. Siegel, and R. Beigel, "Plaque disruption during transcatheter aortic valve replacement," Eur. Heart J. Cardiovasc. Imaging, vol. 15, no. 1, p. 117, jan 2014.

[5] J. Bonatti, G. Vetrovec, C. Riga, O. Wazni, and P. Stadler, "Robotic technology in cardiovascular medicine," Nature Reviews Cardiology, vol. 11, no. 5, pp. 266-275, mar 2014.

[6] H. Rafii-Tari, C. J. Payne, and G.-Z. Yang, "Current and Emerging Robot-Assisted Endovascular Catheterization Technologies: A Review," Annals of biomedical engineering, vol. 42, no. 4, pp. 697-715, 2014.

[7] S. Kesner and R. Howe, "Position control of motion compensation cardiac catheters," Robotics, IEEE Transactions on, vol. 27, no. 6, pp. 1045-1055, 2011.

[8] —, "Robotic catheter cardiac ablation combining ultrasound guidance and force control," The International Journal of Robotics Research, 2014.

[9] G. J. Vrooijink, T. T. Ellenbroek, P. Breedveld, J. G. Grandjean, and S. Misra, "A preliminary study on using a robotically-actuated delivery sheath (rads) for transapical aortic valve implantation," in IEEE Int. Conf. Robot. Autom. (ICRA'14), 2014, pp. 4380-4386.

[10] Y. Fu, H. Liu, W. Huang, S. Wang, and Z. Liang, "Steerable catheters in minimally invasive vascular surgery," The International Journal of Medical Robotics and Computer Assisted Surgery, vol. 5, no. 4, pp. 381-391, 2009.

[11] K. Ikuta, H. Ichikawa, K. Suzuki, and D. Yajima, "Multi-degree of freedom hydraulic pressure driven safety active catheter," in Robotics and Automation, 
2006. ICRA 2006. Proceedings 2006 IEEE International Conference on, May 2006, pp. 4161-4166.

[12] A. Devreker, E. Vander Poorten, P. Tran, H. De Praetere, P. Herijgers, J. Vander Sloten, and D. Reynaerts, "Towards fluidic actuation for catheter-based interventions," in Proceedings Actuator 2014, Bremen, Germany, 2014, pp. 173-176.

[13] J. Jayender, R. V. Patel, and S. Nikumb, "Robotassisted active catheter insertion: algorithms and experiments," The International Journal of Robotics Research, vol. 28, no. 9, pp. 1101-1117, 2009.

[14] L. Swanstrom and B. Zheng, "Spatial Orientation and Off-Axis Challenges for NOTES," Gastrointestinal Endoscopy Clinics of North America, vol. 18, no. 2, pp. 315-324, Apr. 2008.

[15] W. Webster, "Omni-directional steerable catheter," 2000, uS Patent 6,123,699.

[16] W. McCoy, "Steerable and aimable catheter," Sep. 24 1985, uS Patent 4,543,090.

[17] C. V. Riga, C. D. Bicknell, R. Sidhu, F. Cochennec, P. Normahani, P. Chadha, E. Kashef, M. Hamady, and N. J. W. Cheshire, "Advanced catheter technology: is this the answer to overcoming the long learning curve in complex endovascular procedures." European journal of vascular and endovascular surgery : the official journal of the European Society for Vascular Surgery, vol. 42, no. 4, pp. 531-8, oct 2011.

[18] M. Csencsits, B. A. Jones, W. McMahan, V. Iyengar, and I. D. Walker, "User interfaces for continuum robot arms," in Intelligent Robots and Systems, 2005.(IROS 2005). 2005 IEEE/RSJ International Conference on. IEEE, 2005, pp. 3123-3130.

[19] R. J. Hendrick, C. R. Mitchell, S. D. Herrell, and R. J. Webster, "Hand-held transendoscopic robotic manipulators: A transurethral laser prostate surgery case study," The International Journal of Robotics Research, pp. 1-14, Jul. 2015.

[20] A. Majewicz and A. M. Okamura, "Cartesian and joint space teleoperation for nonholonomic steerable needles," in 2013 World Haptics Conference (WHC). IEEE, Apr. 2013, pp. 395-400.

[21] L. H. Kim, C. Bargar, Y. Che, and A. M. Okamura, "Effects of master-slave tool misalignment in a teleoperated surgical robot," in 2015 IEEE International Conference on Robotics and Automation (ICRA). IEEE, May 2015, pp. 5364-5370.

[22] H. Wang and Y. Xie, "Passivity based task-space bilateral teleoperation with time delays," in Robotics and Automation (ICRA), 2011 IEEE International Conference on. IEEE, 2011, pp. 2098-2103.

[23] H.-S. Yoon and B.-J. Yi, "Design of a master device for controlling multi-moduled continuum robots," Proceedings of the Institution of Mechanical Engineers, Part C: Journal of Mechanical Engineering Science, vol. 0, no. 0, pp. 1-11, dec 2015.

[24] C. Truckai, "Shapable handle for steerable electrode catheter," Mar. 14 1995, uS Patent 5,397,304.

[25] P. Breedveld, J. Sheltes, E. M. Blom et al., "A new, easily miniaturized steerable endoscope," Engineering in Medicine and Biology Magazine, IEEE, vol. 24, no. 6, pp. 40-47, 2005.

[26] P. Breedveld, "Steerable laparoscopic cable-ring forceps," Journal of Medical Devices, vol. 4, no. 2, p. 027518, 2010.

[27] B. Rosa, A. Devreker, H. De Praetere, C. Gruijthuijsen, S. Portoles-Diez, A. Gijbels, D. Reynaerts, P. Herijgers, J. Vander Sloten, and E. Vander Poorten, "Intuitive Teleoperation of Active Catheters for Endovascular Surgery," in International Conference on Intelligent Robots and Systems - IROS, Hamburg, 2015, pp. 2617-2624.

[28] T. Alderliesten, M. K. Konings, and W. J. Niessen, "Modeling friction, intrinsic curvature, and rotation of guide wires for simulation of minimally invasive vascular interventions." IEEE transactions on bio-medical engineering, vol. 54, no. 1, pp. 29-38, Jan. 2007.

[29] T. C. Gasser, R. W. Ogden, and G. A. Holzapfel, "Hyperelastic modelling of arterial layers with distributed collagen fibre orientations," Journal of The Royal Society Interface, vol. 3, no. 6, pp. 15-35, Feb. 2006.

[30] D. a. Vorp, B. J. Schiro, M. P. Ehrlich, T. S. Juvonen, M. Ergin, and B. P. Griffith, "Effect of aneurysm on the tensile strength and biomechanical behavior of the ascending thoracic aorta," The Annals of Thoracic Surgery, vol. 75, no. 4, pp. 1210-1214, Apr. 2003.

[31] K. Kazmierska, M. Szwast, and T. Ciach, "Determination of urethral catheter surface lubricity," Journal of Materials Science: Materials in Medicine, vol. 19, no. 6, pp. 2301-2306, 2008.

[32] G. Smoljkic, C. Gruijthuijsen, J. Vander Sloten, and E. Vander Poorten, "Towards Intraoperative Use of Surgical Simulators : Evaluation of Catheter Insertion Models," in Proceeding of the 3rd Joint Workshop on New Technologies for Computer/Robot Assisted Surgery (CRAS), 2013, pp. 45-47.

[33] A. Gijbels, E. B. Vander Poorten, P. Stalmans, H. Van Brussel, and D. Reynaerts, "Design of a teleoperated robotic system for retinal surgery," in 2014 IEEE International Conference on Robotics and Automation (ICRA). IEEE, May 2014, pp. 2357-2363.

[34] R. Liao, Y. Tan, H. Sundar, M. Pfister, and A. Kamen, "An Efficient Graph-Based Deformable 2D/3D Registration Algorithm with Applications for Abdominal Aortic Aneurysm Interventions," in Medical Imaging and Augmented Reality, ser. Lecture Notes in Computer Science. Berlin, Heidelberg: Springer Berlin Heidelberg, 2010, vol. 6326, pp. 561-570.

[35] C. Shi, S. Giannarou, S.-l. Lee, and G.-z. Yang, "Simultaneous catheter and environment modeling for Trans-catheter Aortic Valve Implantation," in 2014 IEEE/RSJ International Conference on Intelligent Robots and Systems, no. Iros. IEEE, sep 2014, pp. 2024-2029.

[36] G.-A. Turgeon, G. Lehmann, G. Guiraudon, M. Drangova, D. Holdsworth, and T. Peters, "2D-3D registration of coronary angiograms for cardiac proce- 
dure planning and guidance." Medical physics, vol. 32, no. 12, pp. 3737-3749, 2005.

[37] P. A. Noseworthy, Z. J. Malchano, J. Ahmed, G. Holmvang, J. N. Ruskin, and V. Y. Reddy, "The impact of respiration on left atrial and pulmonary venous anatomy: Implications for image-guided intervention," Heart Rhythm, vol. 2, no. 11, pp. 1173-1178, nov 2005.

[38] T. Heimann, P. Mountney, M. John, and R. Ionasec, "Real-time ultrasound transducer localization in fluoroscopy images by transfer learning from synthetic training data," Medical Image Analysis, vol. 18, no. 8, pp. 1320-1328, 2014.

[39] M. Quigley, K. Conley, B. Gerkey, J. FAust, T. Foote, J. Leibs, E. Berger, R. Wheeler, and A. Mg, "ROS: an open-source Robot Operating System," in ICRA workshop on open source software, vol. 3, 2009, p. 5.

[40] H. Bruyninckx, P. Soetens, and B. Koninckx, "The real-time motion control core of the Orocos project," in IEEE International Conference on Robotics and Automation, 2003, pp. 2766-2771.

[41] P. Soetens, "A software framework for real-time and distributed robot and machine control," Ph.D. dissertation, Department of Mechanical Engineering, Katholieke Universiteit Leuven, Belgium, May 2006.

[42] W. Schroeder, K. Martin, and B. Lorensen, The Visualization Toolkit, 4th ed. Kitware, 2006.

[43] L. Dagum and R. Menon, "Openmp: an industry standard api for shared-memory programming," Computational Science \&6 Engineering, IEEE, vol. 5, no. 1, pp. 46-55, 1998.

[44] S. G. Hart, "Nasa-task load index (nasa-tlx); 20 years later," in Proceedings of the human factors and ergonomics society annual meeting, vol. 50, no. 9. Sage Publications, 2006, pp. 904-908.

[45] M. G. Pellen, L. F. Horgan, J. R. Barton, and S. E. Attwood, "Construct validity of the promis laparoscopic simulator," Surgical endoscopy, vol. 23, no. 1, pp. 130-139, 2009.

[46] K. Tanoue, M. Uemura, H. Kenmotsu, S. Ieiri, K. Konishi, K. Ohuchida, M. Onimaru, Y. Nagao, R. Kumashiro, M. Tomikawa et al., "Skills assessment using a virtual reality simulator, lapsim, after training to develop fundamental skills for endoscopic surgery," Minimally Invasive Therapy \& Allied Technologies, vol. 19, no. 1, pp. 24-29, 2010.

[47] P. Kanumuri, S. Ganai, E. M. Wohaibi, R. W. Bush, D. R. Grow, and N. E. Seymour, "Virtual reality and computer-enhanced training devices equally improve laparoscopic surgical skill in novices," JSLS, Journal of the Society of Laparoendoscopic Surgeons, vol. 12, no. 3, pp. 219-226, 2008.

[48] S. Balasubramanian, A. Melendez-Calderon, and E. Burdet, "A robust and sensitive metric for quantifying movement smoothness," IEEE Trans. Biomed. Eng., vol. 59, no. 8, pp. 2126-2136, 2012.

[49] S. J. Estrada, "Quantitative movement analysis in endovascular surgical tasks for objective determination of skill," Ph.D. dissertation, Doctoral Thesis, Rice
University, 2014.

[50] K. Lesack and C. Naugler, "An open-source software program for performing bonferroni and related corrections for multiple comparisons," Journal of pathology informatics, vol. 2, 2011.

[51] W. Guo, "A note on adaptive bonferroni and holm procedures under dependence," Biometrika, p. asp048, 2009.

[52] V. Strbac, J. Vander Sloten, and N. Famaey, "Analyzing the potential of GPGPUs for real-time explicit finite element analysis of soft tissue deformation using CUDA," Finite Elements in Analysis and Design, vol. 105, pp. 79-89, nov 2015.

[53] M. Coylewright, A. K. Cabalka, J. A. Malouf, J. B. Geske, P. M. Pollak, R. M. Suri, and C. S. Rihal, "Percutaneous mitral valve replacement using a transvenous, transseptal approach: Transvenous mitral valve replacement," JACC: Cardiovascular Interventions, vol. 8, no. 6, pp. 850-857, 2015.

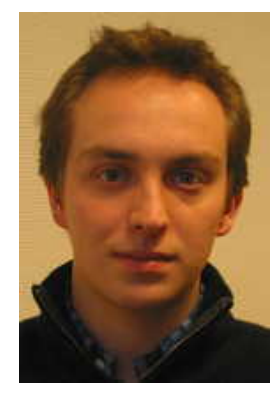

Alain Devreker received the M.Sc. degree in Electro-mechanical Engineering from the Université catholique de Louvain, Louvain-la-Neuve, Belgium, in 2012. He currently is a Ph.D. researcher within the Robot Assisted Surgery group at the Mechanical Engineering Department of KU Leuven, Belgium. His research interests include mechatronic design, control and experimental validation of continuum robots for minimally invasive surgical applications.

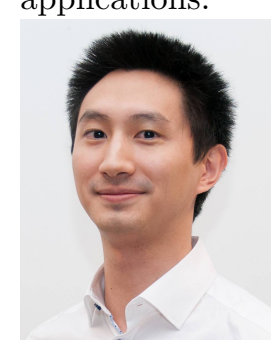

Phuong Toan Tran received the M.Sc. degree in Biomedical Engineering from the Université Libre de Bruxelles, Brussels, Belgium, in 2012. He currently is a Ph.D. researcher within the Robot Assisted Surgery group at the Mechanical Engineering Department of KU Leuven, Belgium. His research interests include sensing, control and virtual reality simulation of continuum robots for minimally invasive surgical applications. 


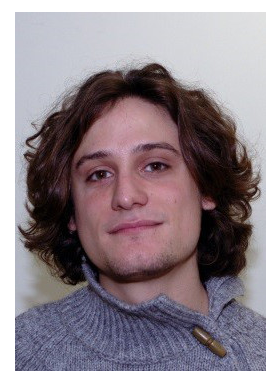

Benoît Rosa received the Engineering degree from Ecole Centrale Paris, France, and the Ph.D. degree in robotics from Pierre et Marie Curie University, Paris, in 2009 and 2013, respectively. From 2013 to 2015, he was a Postdoctoral Researcher within the Robot Assisted Surgery group, Mechanical Engineering Department, KU Leuven, Belgium. Since September 2015, he is with the Pediatric Cardiac Bioengineering group, Boston Childrens Hospital, Harvard Medical School. His research interests include design and control of robotic devices for minimally invasive surgery, flexible actuation systems, and image-guided interventions.

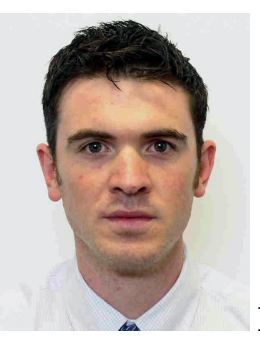

Herbert De Praetere received his bachelor and master degree from KU Leuven, Belgium in 2000 and 2004 respectively. He obtained his degree of general surgeon in 2010 and finished his further specialization of cardiac surgery in 2012. In 2012 he started his PhD titled: "The influence of minimal access surgery on biological outcome: in search of involved pathophysiological pathways." Since 2012 he was working as a staff member in the university hospital of Leuven. Since 2015 he is working also in the general hospital of Bonheiden as a cardiac surgeon. In both hospitals he takes a special interest in minimal invasive techniques, especially transcutaneous valves and robotic enhanced techniques.

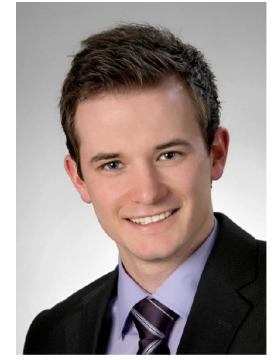

Nicolai Häni received his B.S and M.S from the Zurich University of Applied Sciences, Zürich, in 2012 and 2015 respectively. His research interests include computer vision with application in robotics, software engineering, algorithms and automated control.

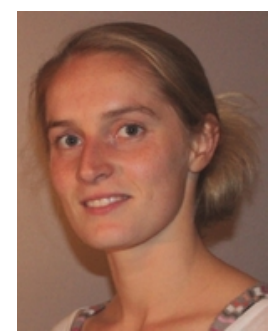

Nele Famaey received the MSc and $\mathrm{PhD}$ degree in mechanical engineering at KU Leuven, Belgium, in 2006 and 2012, respectively. From 2012 on, she is a postdoctoral research fellow of the Flemish research fund (FWO) at the Department of Mechanical Engineering, KU Leuven, where she coordinates the research on soft tissue biomechanics. Her research interests include nonlinear finite element modeling of biological soft tissue, experimental characterization of material properties of cardiovascular tissue, damage detection and quantification in cardiovascular tissue and computational aspects of robotic surgery.

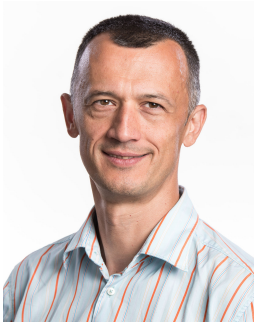

Dejan Šeatović received the engineering degree from Karlsruhe Institute of Technology (KIT), Karlsruhe, Germany in 2000. He obtained the PhD degree in Geomatics from from Leibniz University Hannover, Hannover, Germany in 2013. He coordinates multiple industry and scientific projects in areas of precision farming, surveying, computer vision, quality assessment and medical technology. He is currently Team Leader of Vision \& Navigation team on ZHAW and he is appointed professor for Engineering Geodesy and Photogrammetry on Karlsruhe University of Applied Sciences. His research areas are computer vision in $2 \mathrm{D}$ and $3 \mathrm{D}$, machine perception, software design and implementation for complex systems and navigation algorithms for autonomous mobile robots.

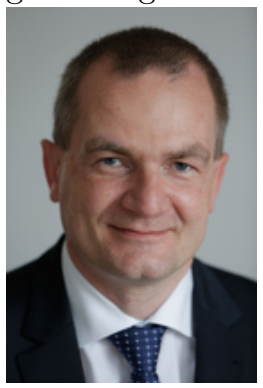

Paul Herijgers obtained his MD in 1991 and PhD in 1998 from KU Leuven, Belgium. He is a board certified cardiac surgeon, practicing in the University Hospital Leuven. He is the chairman of the Department of Cardiovascular Sciences of KU Leuven, covering biomedical cardiovascular research, from basic over translational to clinical research. His research interest is focused on myocardial protection and minimal access heart valve surgery, 
including new enabling technologies.

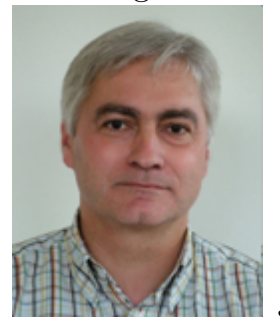

Jos Vander Sloten obtained his M.S. and Ph.D. in Mechanical Engineering from KU Leuven in 1985 and 1990, respectively. Currently he is full professor at the Biomechanics Section, Department of Mechanical Engineering at KU Leuven. He also chairs the Leuven Medical Technology Centre (L-MTC). His teaching assignments are engineering mechanics, problem solving and engineering design, computer integrated surgery systems. His research interests are computer applications in musculoskeletal biomechanics and computer integrated surgery, on which he authored more than 160 journal papers. He is member of the council of the Belgian Society for Medical and Biological Engineering and Computing, and a former council member of the European Society of Biomechanics. In the European Alliance for Medical and Biological Engineering and Science (EAMBES) he served as secretary-general (20032004), president-elect (2005) and president (2006). He was elected Founding Fellow of EAMBES. He is a co-founder of the spin-off company Custom8, member of the board of directors of the company Materialise NV and provides consultancy to the company Mobelife NV.

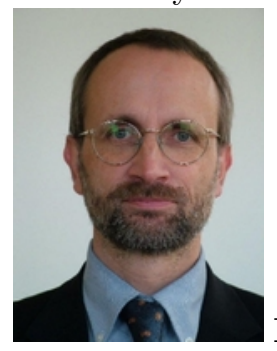

Dominiek Reynaerts obtained an M.S. degree (1986) and a Ph.D. degree (1995) in Mechanical Engineering from KU Leuven. He is a Full Professor in micro-and precision engineering at the KU Leuven, Department of Mechanical Engineering and he is since 2008 also chairing this department with a total staff of about 320 people. His major research interests include manufacturing and machine design with focus on precision engineering and micromechanical actuation systems, with main applications in medical robotics and instrumentation, machine tools and metrology, and energy systems. He is member of IEEE, EUSPEN - European Society of Precision Engineering and Nanotechnology - and of EFFRA - European Factories of the Future Research Association.

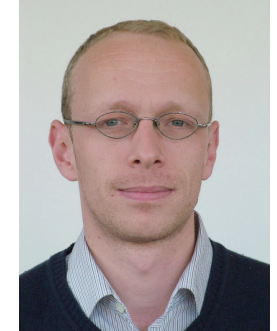

Emmanuel B. Vander Poorten received his B.S and M.S degree from the University of Leuven, Belgium and his Ph.D. degree from the University of Kyoto, Japan, all in Mechanical Engineering in 1998, 2000, and 2007, respectively. From 2007 to 2014, he was a Postdoctoral Researcher at the University of Leuven, Belgium. From 2015, he joined the faculty at the Department of Mechanical Engineering, Belgium, where he is now an Assistant Professor. His research interests include design and control of haptic interfaces, surgical robots, surgical instruments and surgery training systems. 